hep-th/0608157

\title{
A Note on (Meta)stable Brane Configurations in MQCD
}

\author{
I. Bena ${ }^{(1)}$, E. Gorbatov ${ }^{(2)}$, S. Hellerman ${ }^{(1)}$, N. Seiberg ${ }^{(1)}$, and D. Shih ${ }^{(3)}$ \\ (1) School of Natural Sciences, Institute for Advanced Study \\ Einstein Dr., Princeton, NJ 08540 \\ (2) Department of Physics, University of California San Diego \\ 9500 Gilman Drive, La Jolla, CA 92093 \\ ${ }^{(3)}$ Department of Physics, Princeton University \\ Princeton, NJ 08540
}

\begin{abstract}
We examine the M-theory version of SQCD which is known as MQCD. In the IIA limit, this theory appears to have a supersymmetry-breaking brane configuration which corresponds to the meta-stable state of $\mathcal{N}=1 S U\left(N_{c}\right)$ SQCD. However, the behavior at infinity of this non-supersymmetric brane construction differs from that of the supersymmetric ground state of MQCD. We interpret this to mean that it is not a meta-stable state in MQCD, but rather a state in another theory. This provides a concrete example of the fact that, while MQCD accurately describes the supersymmetric features of SCQD, it fails to reproduce its non-supersymmetric features (such as meta-stable states) not only quantitatively but also qualitatively.
\end{abstract}

August 2006 


\section{Introduction}

Stringy versions of quantum field theories have proved to be an important tool in giving an intuitive geometric understanding of gauge theory dynamics. In particular, one can construct supersymmetric configurations of D-branes and NS5 branes in type IIA string theory whose low energy spectrum matches that of a wide variety of fourdimensional supersymmetric gauge theories [1 1 -3]. We will refer henceforth to the general, nonrenormalizable type IIA stringy embeddings of these field theories as the parameter space of MQCD theories. For generic values of the parameters, the correct description of MQCD is in terms of objects in M-theory, such as M5 branes. But MQCD also has two interesting $g_{s} \rightarrow 0$ limits that we will consider in this paper.

The first limit is when $g_{s}$ is infinitesimal and all the length scales of the system are larger than $\sim \ell_{s}$. This we will call the "D-brane limit," because in this limit the dynamics of the system may usefully be described by the Born-Infeld action of a set of D-branes moving in a flat background. The interaction of the dynamical D-branes of interest with other background branes is given by boundary conditions on the dynamical D-branes, and by the introduction of additional, bifundamental degrees of freedom charged under various gauge fields. The degrees of freedom in this limit are the open strings where both $g_{s}$ and $\alpha^{\prime}$ corrections can be treated as small.

The second limit is one in which $g_{s}$ is infinitesimal, but various length scales also scale to zero as $g_{s} \ell_{s}$. This we will refer to as the "decoupling limit" or "SQCD limit," because in this limit one obtains an interacting gauge theory on the D-branes, decoupled from all other stringy degrees of freedom.

For theories with $\mathcal{N}=1$ supersymmetry in 4 dimensions, the decoupling limit and Dbrane limit of MQCD theories generally do not overlap. In general the string embeddings are coupled to a large number of modes above and beyond the modes of the $4 \mathrm{D}$ field theory one wants to study. Bulk gravity modes, Kaluza-Klein modes of higher-dimensional field theories, light modes on nearby branes, string oscillator modes and other degrees of freedom interact with the degrees of freedom of the 4D field theory of interest. In the D-brane limit, these massive modes have not decoupled, so the field theory description is not valid. Conversely, when the field theory description is applicable, one cannot view the system in terms of classical D-branes, since these branes are extended over sub-stringy distances.

Although these two limits lead to different theories, holomorphy ensures that supersymmetric quantities calculated both in the two limits or in other points of the MQCD 
parameter space can be smoothly continued to any other point of the MQCD parameter space [46]. On the other hand, non-supersymmetric quantities are not protected by holomorphy, and so they cannot in principle be continued from one limit to another. Nevertheless, one might have hoped that non-supersymmetric features of the dynamics should, at least qualitatively, be independent of one's precise location in the parameter space of MQCD. In this paper, we will find a concrete counterexample to this, in the specific context of $\mathcal{N}=1 S U\left(N_{c}\right)$ SQCD and its extension to MQCD.

Recently, it has been shown that massive $\mathcal{N}=1 S U\left(N_{c}\right)$ SQCD in the free-magnetic phase exhibits dynamical supersymmetry breaking in meta-stable vacua [7]. This phenomenon was subsequently demonstrated in many similar theories 8-15. One might hope to find such meta-stable vacua at other points in the MQCD parameter space. Instead, we will show in this paper that there is a simple obstruction to continuing the meta-stable vacuum of SQCD into MQCD.

The obstruction has to do with the phenomenon of brane bending. At tree-level in $g_{s}$, the NS5-branes in the brane construction are straight. However, because of string interactions they curve [2]. The direction of this bending is determined by the charges and directions of the D-branes which end on the NS5-brane. Therefore, a proper way to define the theory on the branes at $g_{s} \neq 0$ is not in terms of the detailed positions of the branes, but instead, in terms of the asymptotic behavior of the branes that stretch to infinity. These are the boundary conditions on the system, while the branes in the interior are dynamical and free to adjust themselves. The supersymmetric brane configuration leads to a specific bending of the NS5-branes, and this bending defines a point in the MQCD parameter space. Every state of this theory, stable or meta-stable must have these boundary conditions at infinity. If it has different boundary conditions it is not a state of the same system.

Now that we understand how to define the system using its correct asymptotic behavior, we can check how the nonsupersymmetric brane configuration bends at infinity. The main result of our investigation is that, at $g_{s} \neq 0$, the putative nonsupersymmetric brane configuration cannot bend at infinity according to the proper boundary conditions. The only solutions of the minimal area equations (or of the equations coming from the NS5 brane worldvolume action) bend in the wrong direction. Hence, they differ by an infinite amount from the supersymmetric brane configurations. These solutions therefore do not correspond to meta-stable MQCD vacua. 
Our results indicate that qualitative nonsupersymmetric features of SQCD, like the existence of a meta-stable vacuum, are not seen in the brane description of this theory. While our analysis does not prove that such meta-stable vacua cannot be seen in brane constructions of other four-dimensional gauge theories, the fact that the simplest example of a gauge theory meta-stable vacuum does not carry over to MQCD seems to indicate that this problem is generic.

It would be most interesting to explore if these kinds of meta-stable vacua exist in other string-theoretic dual descriptions of gauge theories, like the gauge-gravity duality. Another interesting application is to "T-dual" versions of the brane constructions. These dualize to branes near a Calabi-Yau singularity that preserves $\mathcal{N}=1$ supersymmetry [16]. It would be interesting to see whether the subtleties we uncover here also appear in this description.

The outline of our paper is as follows. We begin in section 2 by reviewing the old brane constructions of massless $\mathcal{N}=1 S U\left(N_{c}\right)$ SQCD and its magnetic dual. The two brane constructions are obtained as two singular limits of the parameter space of MQCD (see figure 1). Different nonoverlapping regions of the diagram are associated with different simple descriptions of the system. The decoupling limit corresponds to the immediate vicinity of the origin where $\Delta L \rightarrow 0$. The magnetic brane configuration is obtained for fixed and positive value of $\Delta L$, while the electric brane configuration is obtained for fixed and negative $\Delta L$.

We then go on to consider the system at nonzero mass. Here the situation is quite different and is shown in figure 2 - turning on a mass yields a SUSY brane configuration in the electric phase but not in the magnetic phase. This parallels the fact that there is a treelevel SUSY vacuum in massive SQCD, but not in its magnetic dual. In the magnetic phase (as in the field theory), the system becomes tachyonic. We follow the tachyon condensation and obtain a pseudo-moduli space of minimal-energy, SUSY-breaking brane configurations. We show that these brane configurations have the right properties to correspond to the tree-level SUSY-breaking vacua of the magnetic dual to massive SQCD [17].

In section 3 we analyze the possibility of lifting these non-supersymmetric configurations to M-theory. To set up the problem, we first review known results on M-theory lifts of the supersymmetric brane configurations. These lift to M5 branes wrapping holomorphic curves in Taub-NUT $\times \mathbb{R}^{2}$. We describe how at zero mass, both the electric and magnetic brane setups lift to the same holomorphic curve in M-theory. Equivalently, we can take two different limits of the same curve in M-theory and find the two different brane setups 


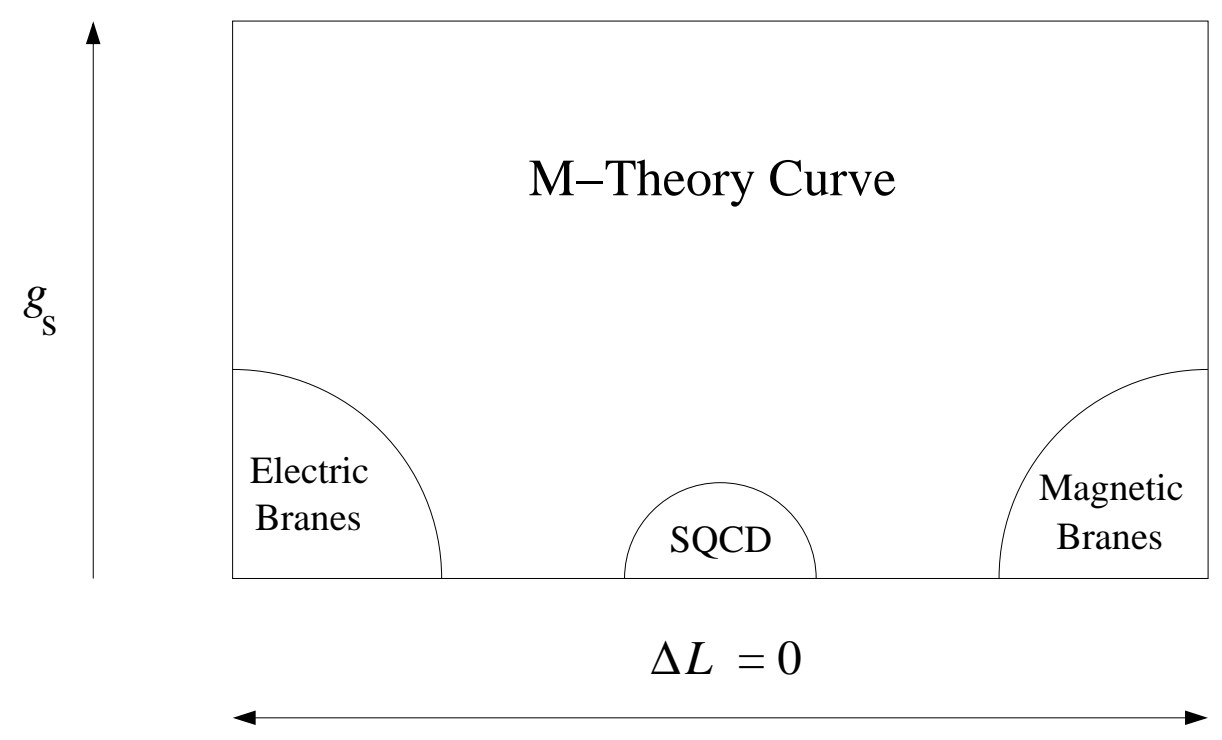

Figure 1: A schematic description of the MQCD parameter space for $m=0$. At small $g_{s}$, there are two "brane limits" - one where $\Delta L$ is fixed and negative, another where $\Delta L$ is fixed and positive as $g_{s} \rightarrow 0$. The SQCD decoupling limit is obtained by taking $\Delta L \rightarrow 0$ and $g_{s} \rightarrow 0$ with $g_{Y M}^{2} \sim g_{s} \ell_{s} / \Delta L$ fixed. These three limits do not overlap with one another. Since the $4 D$ gauge coupling vanishes in both brane limits, there can be no duality connecting them directly.

[18,19]. This is the sense in which the electric-magnetic duality of [17] is implemented in this context. At nonzero mass, we explain how a smooth supersymmetric M-theory curve exists for every nonzero value of the mass parameter, but - unlike the situation for zero mass - there is no smooth limit which corresponds to a magnetic brane configuration, and only the limit to the electric brane configuration is well-defined (see figure 2).

We then tackle the problem of lifting the tree-level SUSY-breaking brane configurations of section 2 to M-theory. Such a configuration should lift to M5 branes wrapping non-holomorphic minimal-area curves in Taub-NUT $\times \mathbb{R}^{2}$. We solve the (rather involved) equations of motion for these curves, and show that there is no solution with the same boundary conditions as the SUSY vacua. In section 4 we undertake a perturbative type IIA analysis of the SUSY-breaking brane configuration at small $g_{s}$, and we find the same result. We conclude from this that, after string interactions are taken into account, there is no meta-stable brane configuration in the D-brane limit of MQCD.

In order to further understand the absence of the metastable vacuum, we analyze in section 5 a kink "quasi-solution" that has the right asymptotic boundary conditions, and 


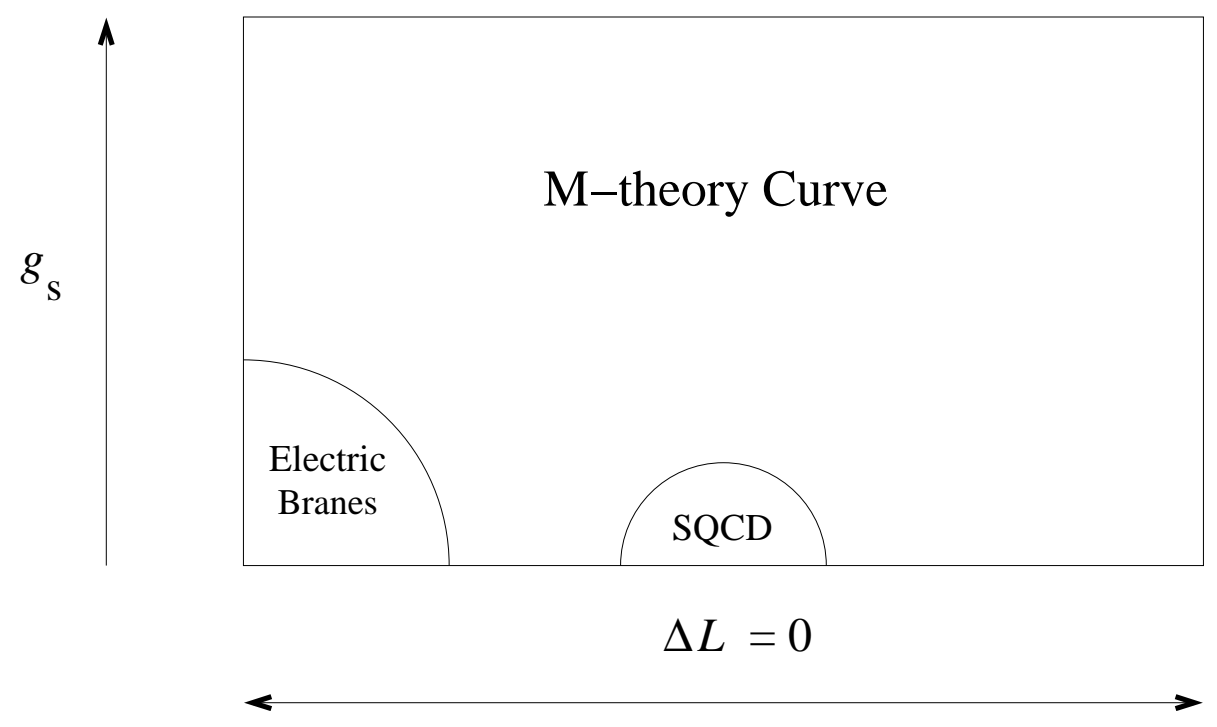

Figure 2: A schematic description of the MQCD parameter space for $m \neq 0$. At small $g_{s}$, there is only one "brane limit" - the one where $\Delta L$ is fixed and negative. The SQCD decoupling limit is again obtained by taking $\Delta L \rightarrow 0$ and $g_{s} \rightarrow 0$ with $g_{Y M}^{2}$ fixed.

reduces to the SUSY-breaking configuration at $g_{s} \rightarrow 0$. We find that this brane "quasisolution" has a runaway mode, which ruins the stability of the solution at nonzero $g_{s}$. Only in the $g_{s} \rightarrow 0$ limit is this runaway mode frozen, and this is the reason the $g_{s}=0$ SUSYbreaking brane configuration is stable. Finally, in appendix A we include some technical details regarding the exact solution of the M5 brane equations of motion.

Note: While this paper was being prepared for publication, two papers which explore the SUSY-breaking brane configuration appeared [20,21]. These results overlap with parts of section 2 of our paper. The authors of [21] also propose a lift of this brane configuration to M-theory, which, as we discuss in more detail in section 3, has different boundary conditions and hence is not a meta-stable MQCD state.

\section{IIA Brane Configurations with $g_{s} \rightarrow 0$}

In this section we review the IIA brane construction of $S U\left(N_{c}\right)$ SQCD and its magnetic dual. Along the way we will clarify a few points that we found confusing in the literature. More details can be found in the review [3] and references therein. 
The brane constructions described in this section ignore the phenomenon of brane bending described in the introduction. As such, they are only capable of describing treelevel physics. To see any effects of string interactions, one must go to M-theory, which is the subject of the next section.

\subsection{Electric configuration}

One can realize massless $\mathcal{N}=1 \mathrm{SQCD}$ with $N_{f}$ flavors and gauge group $S U\left(N_{c}\right)$ on the following set of branes [22]:

- $N_{f}$ D6 branes stretched in the 0123789 directions, at $x^{4,5,6}=0$.

- An NS5 brane in the 012345 directions at $x^{8,9}=0, x^{6}=L_{0}+\Delta L$, with $\Delta L<0$ (the NS brane).

- An NS5 brane in the 012389 directions at $x^{4}=x^{5}=0, x^{6}=L_{0}$ (the NS' brane).

- $N_{f}$ D4 branes running between the D6 brane and the NS brane (the "flavor" D4 branes).

- $N_{c}$ D4 branes running between the NS' brane and the NS brane (the "color" D4 branes).

This brane configuration is depicted in figure 3 .

Let us briefly describe the low-energy effective field theory living on the D4 branes. The massless modes comprise $\mathcal{N}=1 S U\left(N_{c}\right)$ SQCD: the 4-4 strings between the color branes give rise to $4 \mathrm{D} S U\left(N_{c}\right)$ gauge fields; while the $4-4$ strings between the color and flavor branes give rise to $N_{f}$ quarks, massless fields $Q_{f}, \widetilde{Q}_{f}$ transforming in the fundamental of $S U\left(N_{c}\right)$. Note that the quarks are four-dimensional because the 4-4 strings are localized at the intersection of the color and flavor branes.

Of course, the theory on the branes is not precisely $\mathcal{N}=1$ SQCD - there are infinite towers of massive modes with masses $\sim|\Delta L|^{-1}, L_{0}^{-1}$ coming from Kaluza-Klein reduction of the various 4-4 strings, as well as the usual open and closed string states with masses $\sim \ell_{s}^{-1}$. In particular, notice that the 4-4 open strings connecting the flavor branes are all massive (with masses $\sim L_{0}^{-1}$ ) because of the boundary conditions at the D6 and the NS brane. 


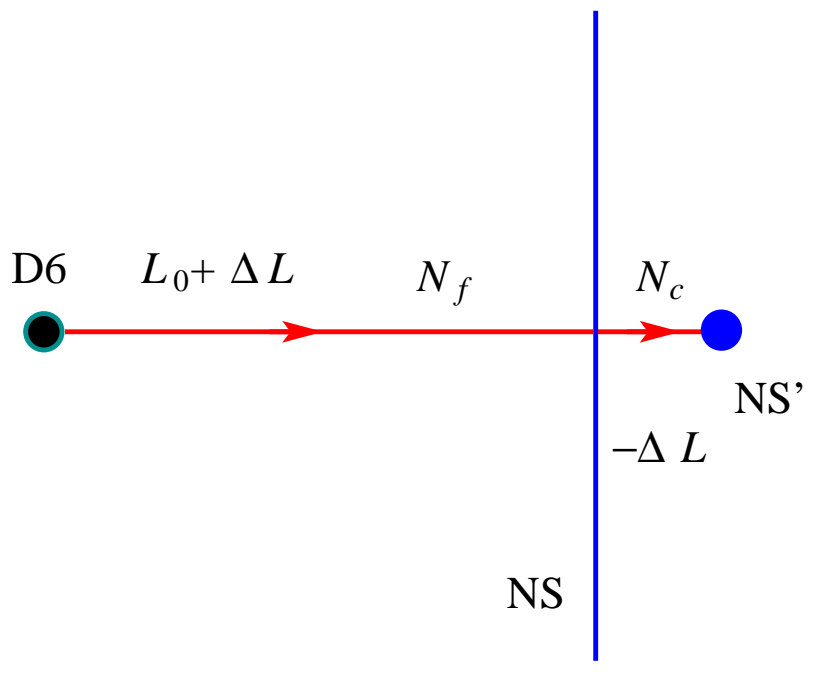

Figure 3: The electric brane configuration describing $S U\left(N_{c}\right) \mathcal{N}=1 S Q C D$. The axes are as follows: the vertical direction represents $\left(x^{4}, x^{5}\right)$, the horizontal direction is $x^{6}$, and coming out of the page are $\left(x^{8}, x^{9}\right)$. All other directions (including the non-compact field theory directions) are suppressed.

If the goal is to decouple the massive states and obtain precisely $S U\left(N_{c}\right)$ SQCD, we should send $\Delta L, L_{0}$ and $\ell_{s} \rightarrow 0$ holding some field theory energy (such as the dynamical scale $\Lambda$ ) fixed. More precisely, in order to obtain a theory with finite 4D gauge coupling, we should take

$$
g_{s} \rightarrow 0, \quad \ell_{s} \rightarrow 0, \quad \Delta L \rightarrow 0
$$

with

$$
\frac{1}{g_{\text {elec }}^{2}}=\frac{|\Delta L|}{g_{s} \ell_{s}}
$$

held fixed. This leaves behind $\mathcal{N}=1 S U\left(N_{c}\right)$ SQCD, with gauge coupling $g=g_{\text {elec }}$.

However, these scaling limits should be interpreted with care. In the limit (2.1), the color D4 branes become extended by only a sub-stringy amount $\Delta L \sim g_{s} \ell_{s}$ deep within the throat of the NS5 branes. So at some point in the decoupling limit, the classical brane picture will break down, and along with it the naive relation (2.2) 1 . But at that point, we should switch over to the description in terms of the decoupled field theory. The upshot is that there is never a regime of parameters where both the flat space string theory and the field theory are simultaneously valid. As discussed in the introduction, we will focus

1 In particular, we expect that (2.2) will receive logarithmic renormalization $\sim \log (|\Delta L| \Lambda)$, which becomes important as $\Delta L \rightarrow 0$. 
in this paper on the regime of parameters where the string theory description is valid. In particular, all the length scales $\left(L_{0}, \Delta L, \ell_{s}\right.$ etc.) will be order one, and massive modes will not have decoupled.

Finally, let us conclude our review of the electric brane configuration by describing a deformation that will be relevant below: moving the D6 branes by an amount $\Delta x$ in the $x^{4}+i x^{5}$ direction. Shown in figure 4 is the supersymmetric configuration resulting from this deformation. As far as the massless modes on the D4 branes are concerned, this deformation corresponds to deforming the superpotential by a mass term for the quarks:2

$$
W=\operatorname{Tr} m Q \widetilde{Q}
$$

The relation between $m$ and $\Delta x$ in the classical brane picture (where one neglects the background geometry sourced by the NS5 branes) is

$$
m=\frac{\Delta x}{\ell_{s}^{2}}
$$

Again, we expect this naive relation to be modified in the field theory decoupling limit (2.1).

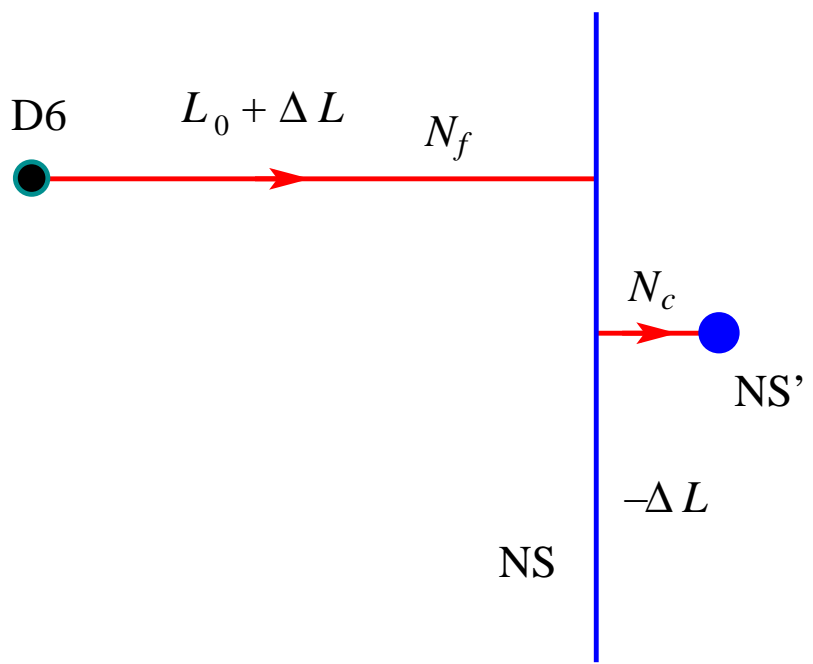

Figure 4: The brane configuration describing the $S U S Y$ vacuum of massive $\mathcal{N}=1 S Q C D$. The axes are the same as in figure 3.

2 Notice also that there is only one SUSY vacuum here; the $N_{c}$ SUSY vacua one expects from the Witten index are only visible after nonperturbative quantum effects are taken into account. This can be done either by studying the gauge theory on the D4 branes, or by going to the M-theory lift. 


\subsection{Magnetic configuration - massless case}

One can realize the magnetic dual to massless $\mathcal{N}=1 S U\left(N_{c}\right)$ SQCD [17] using the following set of branes [22]:

- $N_{f}$ D6 branes stretched in the 0123789 directions, at $x^{4,5,6}=0$.

- An NS5 brane in the 012389 directions at $x^{4}=x^{5}=0, x^{6}=L_{0}$ (the NS' brane).

- An NS5 brane in the 012345 directions at $x^{8,9}=0, x^{6}=L_{0}+\Delta L$, with $\Delta L>0$ (the NS brane)

- $N_{f}$ D4 branes running between the D6 brane and the NS' brane (the "flavor" branes).

- $N_{f}-N_{c}$ D4 branes running between the NS' brane and the NS brane (the "color" branes).

This brane configuration is shown in figure 5. In particular we should note that it has different behavior at infinity compared to the electric brane configuration shown in figure 3. Therefore, it describes a different theory in the parameter space of MQCD.

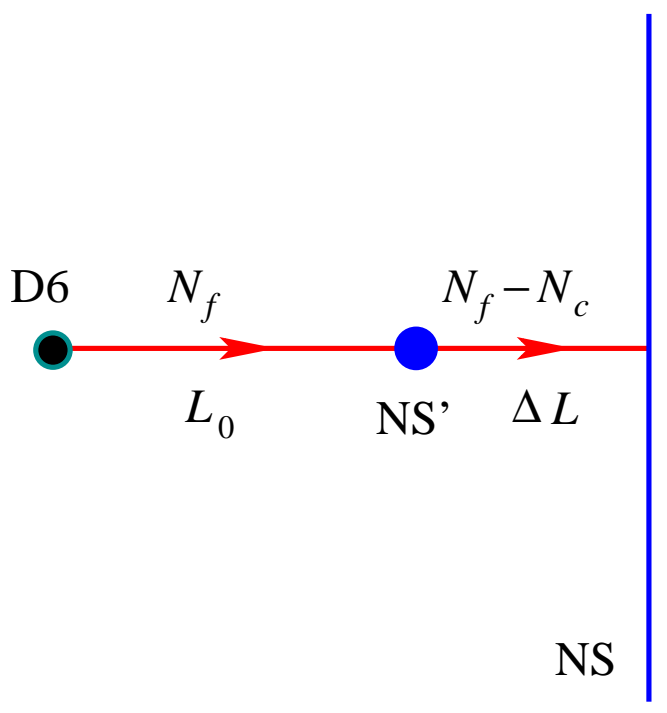

Figure 5: The brane configuration describing the magnetic dual to massless $\mathcal{N}=1 S U\left(N_{c}\right)$ $S Q C D$. The axes are the same as in figure 3.

Now the low-energy effective field theory living on the D4-branes is given by the following. The massless fields are those of the magnetic dual to SQCD [17]: 4-4 strings between the color branes give rise to $4 \mathrm{D} S U\left(N_{f}-N_{c}\right)$ gauge fields; 4-4 strings between the color and flavor branes give rise to the magnetic quarks, i.e. fields $q_{f}, \widetilde{q}_{f}$ transforming in 
the fundamental of $S U\left(N_{f}-N_{c}\right)$; and fluctuations of the 4-4 flavor strings in the $\left(x^{8}, x^{9}\right)$ directions give rise to an $N_{f} \times N_{f}$ matrix of gauge-singlet chiral superfields

$$
w_{f g}=\left(x^{8}+i x^{9}\right)_{f g}
$$

Our conventions are that $q$ and $\widetilde{q}$ are canonically normalized, dimension one fields, while $w$ has mass dimension -1 . These fields have leading-order Kähler potential given by

$$
K=\frac{L_{0}}{g_{s} \ell_{s}^{5}} w^{\dagger} w+q^{\dagger} q+\widetilde{q}^{\dagger} \widetilde{q}+\ldots
$$

coming from the Born-Infeld action on the flavor D4 branes. From the geometry, it is clear that the massless fields interact via the superpotential

$$
W=\frac{1}{\ell_{s}^{2}} \operatorname{Tr} w q \widetilde{q}
$$

where the Yukawa coupling is fixed by the requirement that the magnetic quarks have physical mass $m_{q}=\Delta w / \ell_{s}^{2}$ when the flavor branes are moved by an amount $\Delta w$ in the $x^{8}+i x^{9}$ direction.

Again, there are other modes with masses $\sim(\Delta L)^{-1}, L_{0}^{-1}$ and $\ell_{s}^{-1}$ which are not decoupled. If we want to remove these states, we should send

$$
g_{s} \rightarrow 0, \quad \ell_{s} \rightarrow 0, \quad \Delta L \rightarrow 0, \quad L_{0} \rightarrow 0
$$

with

$$
\frac{1}{g_{m a g}^{2}}=\frac{\Delta L}{g_{s} \ell_{s}}, \quad h=\sqrt{\frac{g_{s} \ell_{s}}{L_{0}}}
$$

held fixed. This leaves behind the magnetic dual to $\mathcal{N}=1 S U\left(N_{c}\right)$ SQCD [17], with gauge coupling $g=g_{m a g}$ and Yukawa coupling $h$. (Note that the Yukawa coupling $h$ is defined in terms of the canonically normalized field $\Phi=\sqrt{\frac{L_{0}}{g_{s} \ell_{s}^{5}}} w$.)

As was the case for the electric brane configuration, the scaling limits should again be interpreted with care. In the limit (2.8), we again expect that the classical brane picture will break down, and the relations (2.9) will be modified. Nevertheless, it is interesting that in the decoupling limits (2.1) and (2.8), $\Delta L \rightarrow 0$, so the electric and magnetic brane configurations appear to become indistinguishable at infinity. This is a hint of electricmagnetic duality, but it is far from a proof, since the brane configurations cannot be trusted in this limit. 


\subsection{Magnetic configuration - massive case}

In the magnetic brane configuration, we can also consider moving the D6 branes by an amount $\Delta x$ in the $x^{4}+i x^{5}$ direction. This has a very different effect than in the electric brane configuration - it breaks supersymmetry, as there is no arrangement of the D4 branes, satisfying charge conservation on the NS5 and D6 branes, which is supersymmetric. The brane configuration one obtains from this deformation is shown in figure 6 . There is an open string tachyon between the color and flavor branes, with mass squared

$$
m_{t a c h}^{2}=-\frac{\Delta x}{\ell_{s}^{2} L_{0}}
$$

Again, we stress that this formula is only valid in the classical brane picture, away from the decoupling limit.

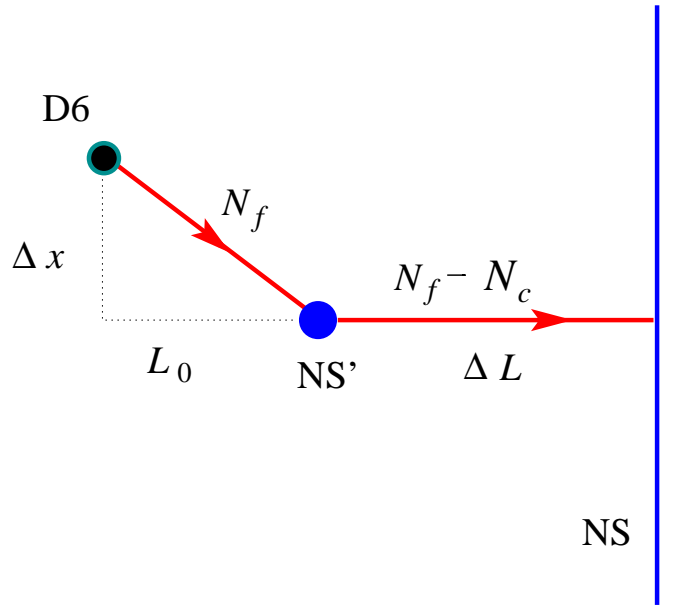

Figure 6: The magnetic brane configuration describing the origin of moduli space in $N_{f}>N_{c}$ massive $\mathcal{N}=1 S Q C D$.

In the field theory living on the D4 branes, the deformation by $\Delta x$ corresponds to adding a term linear in $w$ to the superpotential:

$$
W=\frac{1}{\ell_{s}^{2}} \operatorname{Tr} w q \widetilde{q}+\frac{\Delta x}{g_{s} \ell_{s}^{5}} \operatorname{Tr} w
$$

where the coefficient of the linear term is fixed by matching the mass of the field theory tachyon with that of the open string tachyon (2.10). Indeed, the $w$ F-term contributes a term to the scalar potential of the form

$$
\begin{aligned}
V \supset K_{w \bar{w}}^{-1}\left|F_{w}\right|^{2} & =\left(\frac{L_{0}}{g_{s} \ell_{s}^{5}}\right)^{-1} \operatorname{Tr}\left|\frac{1}{\ell_{s}^{2}} q \widetilde{q}+\frac{\Delta x}{g_{s} \ell_{s}^{5}}\right|^{2} \\
& =\frac{N_{f}|\Delta x|^{2}}{g_{s} \ell_{s}^{5} L_{0}}+\frac{\Delta x}{\ell_{s}^{2} L_{0}}(q \widetilde{q}+\text { c.c. })+\mathcal{O}\left((q \widetilde{q})^{2}\right)
\end{aligned}
$$


We see in the $q \widetilde{q}$ quadratic term precisely the open string tachyon with the requisite mass.

The linear term in (2.11) spontaneously breaks supersymmetry at tree level (and to all orders in perturbation theory) by the rank condition. It is important that the supersymmetry breaking must be spontaneous in the field theory, because the brane configuration has supersymmetric boundary conditions - the only objects that stretch to infinity are the mutually BPS NS5 branes and D6 brane. Notice also that the gauge theory on the branes is expected to have $N_{c}$ SUSY vacua coming from non-perturbative effects in the magnetic gauge group. We cannot see these here, just as we could not see the $N_{c}$ SUSY vacua in the electric brane configuration. As discussed at the beginning of the section, the reason is that the brane configuration only captures the physics that is perturbative in $g_{s}$. Non-perturbative physics is only visible in the M-theory lift, to be discussed in the next section.

As a consistency check, we can compute the vacuum energy density of this (tachyonic) state two ways, first using field theory (2.11), which gives the constant piece in (2.12):

$$
V_{t a c h}=\frac{N_{f}|\Delta x|^{2}}{g_{s} \ell_{s}^{5} L_{0}}
$$

and second with the DBI action, i.e. the length of the D4 branes,

$$
V_{D B I}=\tau_{4}\left(N_{f} \sqrt{|\Delta x|^{2}+L_{0}^{2}}+\left(N_{f}-N_{c}\right)|\Delta L|\right)
$$

In the $\Delta x \ll L_{0}$ limit, and substituting $\tau_{4} \sim 1 / g_{s} \ell_{s}^{5}$ for the D4 brane tension, we find agreement between (2.13) and the $|\Delta x|$ dependent part of (2.14). This is a consistency check of the approximately canonical Kähler potential (2.6).

\subsection{Minimal-energy SUSY-breaking brane configuration}

Since the configuration shown in figure 6 is tachyonic, it can lower its energy by condensing the tachyon. This happens by reconnecting $N_{f}-N_{c}$ of the D4 branes, whereby they snap into the horizontal $x^{6}$ direction. The resulting minimal-energy SUSY-breaking configuration is shown in figure 7. 


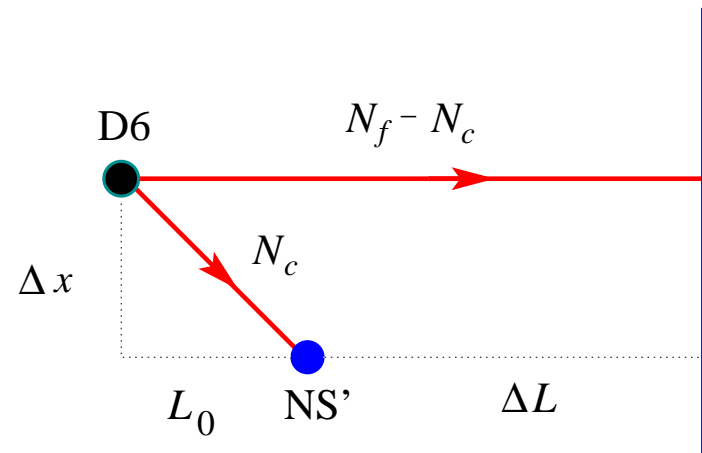

NS

Figure 7: The minimal-energy SUSY-breaking brane configuration.

In the field theory on the D4 branes, the snapping of the D4 branes corresponds to giving $q \widetilde{q}$ an expectation value $\sim \Delta x$, which Higgses the magnetic gauge group. Indeed, since the superpotential and leading-order Kähler potential are algebraically identical to the ones analyzed in [7], one can repeat the calculations in that paper line by line to show that the system described by (2.6) (2.11) has a SUSY-breaking vacuum, in which $\left(N_{f}-N_{c}\right)^{2}$ of the $q$ 's and $\widetilde{q}$ 's have acquired an expectation value.

The brane configuration described in figure 7 is clearly the analogue of the SUSYbreaking vacuum found at tree-level in the magnetic dual to SQCD. 3 In the remainder of this subsection, we will list some consistency checks of this claim.

(1) Energies and energy differences. One obvious check is to compare the energy of the SUSY-breaking vacuum, $V_{0}$, computed using the effective field theory and using the DBI action. As in [7], the former gives

$$
V_{0}=\frac{N_{c}|\Delta x|^{2}}{g_{s} \ell_{s}^{5} L_{0}}
$$

while the latter gives

$$
V_{D B I}=\tau_{4}\left(N_{c} \sqrt{|\Delta x|^{2}+L_{0}^{2}}+\left(N_{f}-N_{c}\right)\left(L_{0}+|\Delta L|\right)\right)
$$

Again, we find complete agreement of the $|\Delta x|$ dependent part, in the limit $|\Delta x| \ll L_{0}$. We can also compare the energy difference between the tachyonic state and the vacuum:

$$
\Delta V_{D B I}=\tau_{4}\left(N_{f}-N_{c}\right)\left(\sqrt{|\Delta x|^{2}+L_{0}^{2}}-L_{0}\right)
$$

3 We should stress, however, the central point of this paper: due to string interactions, this brane configuration is actually not related to the meta-stable state of SQCD. We will elaborate on this in detail in the later sections. 
which agrees exactly with the energy difference computed from the field theory in the $\Delta x \ll L_{0}$ limit.

(2) Symmetries. The SUSY-breaking brane configuration carries the same quantum numbers as the SQCD meta-stable state. First of all, there are no D4 branes between the NS5 branes, so the magnetic gauge group is completely Higgsed. Second, there are two groups of D4 branes, and so the non-Abelian flavor symmetry $S U\left(N_{f}\right)$ is spontaneously broken down to $S U\left(N_{f}-N_{c}\right) \times S U\left(N_{c}\right)$, as in SQCD. Finally, the configuration is invariant under rotations in the 89 plane, so the state preserves the same accidental R-symmetry as in SQCD, under which the mesons have charge 2 and the magnetic quarks charge 0. (For a discussion of the various $U(1)$ factors in the brane configuration and how they match onto the field theory, see e.g. [3], p. 153.)

(3) Pseudo-moduli. The SUSY-breaking brane configuration also has the same noncompact pseudo-moduli as the SQCD meta-stable state. Since the D6 and the NS' are parallel, we can slide the $N_{c}$ D4 branes independently along the 89 directions. These modes correspond to the eigenvalues of the $N_{c} \times N_{c}$ matrix of pseudo-moduli $\delta \Phi_{0}$ in the field theory. They will get a potential at (open-string) one-loop from the interaction with the $N_{f}-N_{c}$ D4 branes; this agrees with the fact that the field theory one-loop potential for $\delta \Phi_{0}$ is proportional to $N_{f}-N_{c}$. This dependence of $N_{f}-N_{c}$ was quite curious from the field theory perspective, but appears obvious from the point of view of the brane construction.

\section{The M-theory Lift}

In the previous section, we constructed a SUSY-breaking configuration of D4, NS5 and D6 branes at infinitesimal $g_{s}$, and we argued that this is an extrapolation of the SUSYbreaking vacuum of SQCD to classical string theory. However, it remains to be seen whether this brane configuration survives the extrapolation to nonzero $g_{s}$, i.e. whether it remains (meta)stable once string interactions are taken into account. In this section, we will analyze the effects of these interactions by lifting our brane configuration to M-theory.

4 More difficult to see in the brane construction are the other pseudo-moduli, as well as the Goldstone bosons from the spontaneously-broken flavor symmetry. Since these correspond to compact field excitations, we expect that they are localized at the singularity where the two stacks of D4 branes meet the D6 branes. 


\subsection{Taub-NUT coordinates}

At nonzero $g_{s}$, the system is described by M-theory on a circle of radius $R=g_{s} \ell_{s}$. The D6 branes, which are extended in the 0123789 directions, lift to Taub-NUT (TN) space with asymptotic radius $R$ and charge $N_{f}$. All the other branes lift to M5 branes extended in the 0123 directions and wrapping various complex curves inside $\mathcal{M}_{6}=\mathrm{TN} \times \mathbb{R}^{2}$, where the second factor represents the 89 directions [4-6]. An M5 brane is supersymmetric if and only if it wraps a complex curve that is holomorphic with respect to a complex structure of $\mathcal{M}_{6}$. Several M5 branes form a supersymmetric configuration if and only if they wrap curves holomorphic with respect to the same complex structure. We will find it convenient at various points to parameterize $\mathcal{M}_{6}$ using two different coordinate systems. Let us now describe these coordinate systems in detail.

The first coordinate system we shall refer to as the "physical coordinates" because they have a natural reduction to IIA. In these coordinates, Taub-NUT is parameterized by $\left(\vec{r}, x^{10}\right)=\left(x^{4}, x^{5}, x^{6}, x^{10}\right)$; and $\mathbb{R}^{2}$ by $\left(x^{8}, x^{9}\right)$ plane. The metric is

$$
d s^{2}=G_{A B} d X^{A} d X^{B}=V d \vec{r}^{2}+V^{-1}\left(d x^{10}+\vec{\omega} \cdot d \vec{r}\right)^{2}+\left(d x^{8}\right)^{2}+\left(d x^{9}\right)^{2}
$$

where

$$
V=1+\frac{N_{f} R}{r}
$$

and

$$
\nabla \times \vec{\omega}=\nabla V
$$

For reasons that will be clear in a moment, we will choose to work in the gauge where

$$
\vec{\omega}=\frac{N_{f} R}{r\left(r+x^{6}\right)}\left(-x^{5}, x^{4}, 0\right)
$$

The second coordinate system we will refer to as the "holomorphic coordinates" because these make explicit the complex Kähler structure of $\mathcal{M}_{6}$. In these coordinates, Taub-NUT is described by complex variables $(v, y)$; and $\mathbb{R}^{2}$ by $w$. Our conventions will be that $(v, y, w)$ have mass dimensions given by

$$
[v]=1, \quad[y]=2 N_{c}, \quad[w]=2
$$

respectively. As described in [23,24] (see also [19] and the appendix of [25]), the explicit change of coordinates between the physical and the holomorphic coordinates is

$$
v=\frac{x^{4}+i x^{5}}{\ell_{s}^{2}} ; \quad y=\mu^{2 N_{c}} e^{\left(x^{6}-L_{0}+i x^{10}\right) / 2 R}\left(\frac{r+x^{6}}{R}\right)^{N_{f} / 2} ; \quad w=\frac{x^{8}+i x^{9}}{R \ell_{s}^{2}}
$$


Here $L_{0}$ is some normalization constant and $\mu$ is some arbitrary dimensionful scale required to give $y$ the correct dimensions. In terms of these, the metric becomes

$$
d s^{2}=G_{i \bar{j}} d X^{i} d X^{\bar{j}}=\left(1+\frac{N_{f} R}{r}\right)\left|\ell_{s}^{2} d v\right|^{2}+R^{2}\left(1+\frac{N_{f} R}{r}\right)^{-1}\left|f \frac{d v}{v}-2 \frac{d y}{y}\right|^{2}+R^{2}\left|\ell_{s}^{2} d w\right|^{2}
$$

where

$$
f=N_{f}\left(1-\frac{x^{6}}{r}\right)
$$

\subsection{Supersymmetric brane configurations in M-theory}

In this subsection, we will review the supersymmetric M5 brane configurations constructed by [5,66]. These are holomorphic with respect to the complex structure described by the coordinates $(v, w, y)$. First we consider the curve that describes the origin of the moduli space of $m=0$ SQCD. It has two components

$$
\begin{aligned}
& \mathcal{C}_{N S}: \quad w(z)=0, \quad v(z)=z, \quad y(z)=\Lambda^{3 N_{c}-N_{f}} z^{N_{f}-N_{c}} \\
& \mathcal{C}_{N S^{\prime}}: \quad w(z)=z, \quad v(z)=0, \quad y(z)=z^{N_{c}}
\end{aligned}
$$

where we have denoted by $\Lambda$ the dynamical scale of the theory. The $\mathcal{C}_{N S}$ component of the curve describes the $N_{f}-N_{c}$ D4 branes ending on the NS brane, while the $\mathcal{C}_{N S^{\prime}}$ component of the curve describes the $N_{c}$ D4 branes ending on the NS' brane.

When the mass is nonzero, the supersymmetric curve has only one component and takes the form:

$$
w=z ; \quad v=m \frac{z+z_{0}}{z} ; \quad y=\frac{\left(z+z_{0}\right)^{N_{f}}}{z^{N_{f}-N_{c}}} .
$$

Here $m$ corresponds to the mass of the electric quarks, while $z_{0}^{N_{c}}=m^{N_{f}-N_{c}} \Lambda^{3 N_{c}-N_{f}}$.

From these formulas, it is straightforward to extract the behavior of the supersymmetric curves in various limits.

1. $v \rightarrow \infty$, leads to

$$
w \rightarrow 0, \quad y \rightarrow \Lambda^{3 N_{c}-N_{f}} v^{N_{f}-N_{c}}+\ldots
$$

We will refer to this as the NS asymptotic region.

2. $w \rightarrow \infty$, leads to

$$
v \rightarrow m, \quad y \rightarrow w^{N_{c}}+\ldots
$$

We will refer to this as the NS' asymptotic region. 
3. In addition, the nature of the map (3.6) between the holomorphic and the physical coordinates requires the condition:

$$
y=0 \quad \text { only if } \quad v=0 .
$$

If this condition were violated, the curve would have a third, unphysical asymptotic region where the M5 branes stretch to $x^{6} \rightarrow-\infty$ (see (3.6)).

For completeness we note that, as mentioned in the introduction, using equation (3.9) for the holomorphic massless curve we can derive two brane configurations: one describing the electric setup, and the other the magnetic setup [18,19]. This is done by writing the two components of the curve (3.9) in terms of physical coordinates (3.6) and taking the limit $R \rightarrow 0$. The same is not true in the case when $m \neq 0$ : the one component curve (3.10) reduces, upon taking $R \rightarrow 0$, to the supersymmetric electric brane configurations, but fails to give the magnetic one.

\subsection{Non-supersymmetric brane configuration - no smooth solution}

Now we are finally ready to tackle the question of whether the non-supersymmetric brane configuration of the previous section survives the continuation to non-zero $g_{s}$. Such a configuration would be an M5 brane wrapping a non-holomorphic, minimal area surface in the (Taub-NUT) $\times \mathbb{R}^{2}$ background, and satisfying the boundary conditions of the previous subsection.

We will have to simplify things by assuming the following ansatz:

A. The curve has two components corresponding to the NS5 and NS5' regions, just as in the case of the $m=0$ supersymmetric curve. These two components touch at the D6 brane (remember, this is at $x^{4,5,6}=0$ ). Only the NS5' component is nonholomorphic and different from the $m=0$ curve. This ansatz is motivated by the picture of the non-supersymmetric IIA brane configuration (see figure 7), where only the NS5' is deformed by turning on $m$. It is also justified by the expectation that the curve should have a singularity at the $\mathrm{D} 6$ brane, since there must be massless modes (the Goldstone bosons and some of the pseudo-moduli) supported there. By contrast, the one-component supersymmetric curve is smooth everywhere, and correspondingly, the supersymmetric vacuum is gapped.

B. $x^{6}+i x^{10}$ with $x^{6} \geq 0$ is a good, global coordinate along the NS' component. Moreover, we will take $m$ to be real without loss of generality, and then we will assume that only 
$x^{4}$ is nonzero along the curve. These assumptions are motivated by the picture of the non-supersymmetric IIA brane configuration, and they greatly simplify the analysis.5

C. Finally, we assume that the only $x^{10}$ dependence of the curve comes in through the phase of $x^{8}+i x^{9}$, as

$$
\arg \left(x^{8}+i x^{9}\right)=\frac{x^{10}}{2 N_{c} R}
$$

This assumption is justified by the $U(1)$ symmetries of the problem. The meta-stable state of SQCD has an accidental $U(1)_{R}$ symmetry that is only broken by the anomaly [7]. In the brane configuration, this $U(1)_{R}$ is identified with rotations in the 89 plane, as discussed above in section 2.4, while the anomaly is identified as a shift in $x^{10}$ under rotations in the 89 plane [4], given precisely by (3.14).

To summarize, our ansatz restricts the form of the non-holomorphic curve to be:

$$
x^{4}=f(s), \quad x^{5}=0, \quad x^{8}+i x^{9}=e^{i x^{10} / 2 N_{c} R} g(s), \quad x^{6}=s
$$

with $s \geq 0$ and $f$ and $g$ real. Now, the action of such a surface is simply

$$
A=\int d^{2} z \sqrt{\operatorname{det} g_{a b}}
$$

where $g_{a b}$ is the $2 \times 2$ induced metric

$$
g_{a b}=\frac{\partial X^{A}}{\partial z^{a}} \frac{\partial X^{B}}{\partial z^{b}} G_{A B}(X)
$$

By substituting (3.15) and (3.1) into the action (3.16) we find the action:

$$
A=\int d s \sqrt{\left(V^{-1}+\frac{g^{2}}{4 R^{2} N_{c}^{2}}\right)\left(V\left(1+f^{\prime 2}\right)+g^{\prime 2}\right)}
$$

where $V$ is the harmonic function of the Taub-NUT space sourced by the $N_{f}$ D6 branes:

$$
V=1+\frac{N_{f} R}{\sqrt{f^{2}+s^{2}}}
$$

5 In fact, one can make even a weaker ansatz that does not assume $x^{6} \geq 0$ is a good coordinate on the entire M-theory curve; see below. 
Using this action we can derive the Euler-Lagrange equations for $f$ and $g$. The equations of motion are quite complicated, and we will not show them here. However, we were able to solve them exactly for $f$; the answer is

$$
f=\frac{c_{2}+c_{3} s+c_{1} \sqrt{\left(c_{2}+c_{3} s\right)^{2}+\left(1-c_{1}^{2}\right) s^{2}}}{1-c_{1}^{2}}
$$

where $c_{1,2,3}$ are integration constants. The derivation of (3.20) is left to appendix A. As a consistency check, note that a straight line $f(s)=a s+b$ is always a solution. Such a line reduces in type IIA to a D4 in the $x^{4}-x^{6}$ plane, which is always BPS with respect to the D6 branes and the NS' brane. So we see that the M-theory equations of motion have correctly reproduced the BPS configurations expected from a IIA analysis.

Now let us apply the boundary conditions $f(s \rightarrow \infty) \rightarrow \Delta x$ and $f(s=0)=0$ to determine the integration constants $c_{1,2,3}$. Here we will run into a problem. The boundary condition at infinity requires $c_{3}=-c_{1}$ and $c_{2}=\Delta x$, but then $f(s=0)=\frac{\Delta x}{1-c_{1}} \neq 0$, so the M5 brane does not end on the D6 brane. Let us illustrate this point with the simplest example, namely $c_{1}=c_{3}=0$ and $f(s)=\Delta x$. Then the equation of motion for $g(s)$ (see appendix A) can be solved exactly; after a straightforward calculation, one finds

$$
g(s)=R \ell_{s}^{2} \mu^{2} e^{\left(s-L_{0}\right) / 2 N_{c} R}\left(\frac{s+\sqrt{(\Delta x)^{2}+s^{2}}}{R}\right)^{N_{f} / 2 N_{c}}
$$

Note that all of the integration constants in (3.21) have been fixed by the boundary condition 2 of section 3.2, i.e. $y \rightarrow w^{N_{c}}$. In fact, in holomorphic coordinates, our solution (3.21) is simply $v=m, y=w^{N_{c}}$ - it is the simplest solution of boundary condition 2 . Unfortunately, it violates boundary condition 3 of section 3.2 , since it does not end on the D6 brane.

In any event, the general solution (3.20) proves a somewhat surprising result: there is no smooth, non-holomorphic M-theory curve obeying the ansatz (3.15), which satisfies the boundary conditions at infinity and ends on the D6 brane at $s=0$ !

Let us understand this conclusion in more detail. For vanishing $g_{s}$ the theory is specified by its brane construction. For nonzero $g_{s}$ this specification of the theory is not precise for two reasons. First, the branes bend at infinity and we should specify the proper boundary conditions. Second, the finite branes in the interior become dynamical and should not be specified; their positions should be set dynamically. Now, let us examine 
the possible boundary conditions for the brane configuration of figure 7 . There are two reasonable options:

1. We can take the same boundary conditions as the asymptotic behavior of the M-theory curve (3.10). This guarantees that we study another state in the same theory. As we showed, there is no solution with these boundary conditions and therefore MQCD does not have such a state.

2. We can let the M-theory lift of the brane configuration of figure 7 bend along the direction of the D4-branes which end on it. Clearly, such an M-theory curve exists. It has two components which preserve different supersymmetries. Hence, the whole curve is not supersymmetric. The state of M-theory which corresponds to this M5brane configuration might or might not be stable. But in any event, since it has different boundary conditions, it is not in the Hilbert space of MQCD.

Let us expand on the second option in more detail. Suppose that instead of first imposing the boundary condition at $s \rightarrow \infty$, we first require the M5 branes to end on the D6 branes $(f(s=0)=0)$. This implies that the solution is a straight line

$$
f(s)=c s
$$

and this will never have the correct asymptotics as $s \rightarrow \infty$. In particular, it will not preserve the same supersymmetries as the NS component of the curve. If $f(s)=c s$, then the equation of motion for $g$ is solved by

$$
g(s)=\frac{1}{\widetilde{\mu}} e^{\sqrt{1+c^{2}}(s-L) / 2 N_{c} R}\left(\frac{s}{R}\right)^{N_{f} / 2 N_{c}}
$$

where $\widetilde{\mu}$ and $L$ are arbitrary (dimensionful) parameters. In fact, if we choose $L=L_{0}$ and $c=\Delta x / L_{0}$, then in the $R \rightarrow 0$ limit, this straight-line solution will reduce to the IIA brane configuration depicted in figure 7. So what we have found is an M-theory lift of the IIA brane configuration with behavior at infinity different than the SUSY curve (3.10). Since different boundary conditions at infinity label different Hilbert spaces, our analysis implies that the SUSY brane configuration and the SUSY-breaking brane configuration are vacua of different theories. This should be contrasted with the field theory, where the SUSY and metastable states are vacua of the same theory.

To conclude this section, let us briefly explain how relaxing the ansatz (3.15) to allow for $x^{6}$ to be of indefinite sign does not help in obtaining a smooth curve corresponding to the meta-stable vacuum. A more general ansatz than (3.15) can be written by taking $w$ 
as the coordinate along the NS' curve. The non-holomorphic NS' component, at general $m$ and $R$, takes the following form:

$$
x^{4}=x^{4}\left(|\widetilde{w}|^{2}\right), \quad x^{5}=0, \quad x^{6}=x^{6}\left(|\widetilde{w}|^{2}\right), \quad x^{10}=2 R N_{c} \arg \widetilde{w}
$$

where $\widetilde{w} \equiv x^{8}+i x^{9}$. Now $x^{6}$ can be both positive and negative; however, we are assuming implicitly that the complex $\widetilde{w}$ plane is a good global cover of the entire NS' component. Substituting this ansatz into the action (3.16), with $z=\widetilde{w}$, we find that $\arg \widetilde{w} \operatorname{drops}$ out, leaving an integral over $\widetilde{r} \equiv|\widetilde{w}|^{2}$. In addition, the action has a global $U(1)$ invariance of rotations in the $\left(x^{4}, x^{6}\right)$ plane. By changing variables to polar coordinates $\left(x^{4}, x^{6}\right) \rightarrow$ $(h(\widetilde{r}), \theta(\widetilde{r}))$, one can show that the action depends only on the derivative of $\theta(\widetilde{r})$ and not on $\theta(\widetilde{r})$ itself. Therefore the variation with respect to $\theta$ produces an integration constant, which can be shown to be $\sim \theta^{\prime 2}$ and vanishes only if $\theta^{\prime}=0$. In fact it is not hard to see that our new ansatz, together with the requirement that the curve ends on the D6 brane, requires this integration constant to be identically zero. Therefore in agreement with previous discussion we conclude that the only solution is a straight line in the $\left(x^{4}, x^{6}\right)$ plane. This again violates the boundary condition that $x^{4} \rightarrow \Delta x$ as $x^{6} \rightarrow \infty$.

\section{IIA Brane Configurations at $g_{s} \neq 0$}

In the previous section, we saw how there was no M-theory lift of the meta-stable state of SQCD. Since the calculations done there involved the classical worldvolume action of the M5 brane, they were, strictly speaking, only valid in the large $R=g_{s} \ell_{s}$ limit. Thus, one might still wonder whether there exists a meta-stable state at small $R$. In this brief section, we will present some heuristic arguments indicating that such a state does not exist even for small $R$.

The analysis is quite similar to the one of the previous section. Instead of using the M5 brane action, we use the effective action of a type IIA NS5 brane, and describe the D4's ending on it as a spike in the NS5 worldvolume.6 We will investigate the SUSY-breaking brane configuration in a regime where $g_{s}$ and $l_{s}$ are small, and $\Delta L, L_{0}$ and $\Delta x$ are all of order one, much larger than $l_{s}$. In this regime the NS5 and D6 are far away and do not considerably influence the dynamics on the NS5' worldvolume.

6 This is very similar to the way in which one describes strings ending on a D-brane using the DBI action [26]. 
Just as at the end the previous section, it will be more convenient here to parameterize the worldvolume of the NS5 brane with $\left(x^{8}, x^{9}\right)$ instead of $\left(x^{6}, x^{10}\right)$. So the relevant part of the NS5' worldvolume Lagrangian [27] is

$$
S=\int d x^{8} d x^{9} g_{s}^{-2} \sqrt{\operatorname{det}\left(g_{m n}+g_{s}^{2} F_{m} F_{n}\right)}
$$

where $m, n$ range over 89 ; and $g_{m n}$ and $F_{m}$ are the induced metric and the one-form field strength, respectively, on the NS5' worldvolume. The field strength is sourced by the $N_{c}$ D4 branes ending on the NS5' brane; in polar coordinates $\left(x^{8}, x^{9}\right) \rightarrow(r, \theta)$, we have

$$
F_{\theta}=N_{c} \ell_{s}
$$

Since the D4 branes are stretched in the 46 directions, we will assume that $x^{4,6,8,9}$ are the only scalars excited on the NS5' worldvolume action. Moreover, we will assume that the solution has radial symmetry, so $x^{M}=x^{M}(r)$. These assumptions stem from the same physical arguments used to justify the M-theory ansatz in subsection 3.3. The Lagrangian now becomes

$$
L=\sqrt{\left(1+\left(\dot{x}^{6}\right)^{2}+\left(\dot{x}^{4}\right)^{2}\right)\left(r^{2}+Q^{2}\right)}
$$

where the dot denotes differentiation with respect to $r$, and

$$
Q=N_{c} g_{s} \ell_{s}
$$

The equations of motion are easily solved for this system; the most general solution is

$$
\begin{aligned}
& x^{4}(r)=c_{3}+c_{1} \log \left(r+\sqrt{r^{2}+Q^{2}-c_{1}^{2}-c_{2}^{2}}\right) \\
& x^{6}(r)=c_{4}+c_{2} \log \left(r+\sqrt{r^{2}+Q^{2}-c_{1}^{2}-c_{2}^{2}}\right)
\end{aligned}
$$

with $c_{1,2,3,4}$ integration constants.

As we have discussed above, our candidate for a meta-stable vacuum should have the same boundary conditions as the brane configuration corresponding to the massive supersymmetric vacuum, because both are vacua of the same theory. It is straightforward to obtain the asymptotic behavior of the supersymmetric vacuum by reducing the massive M-theory curve; the result is (keep in mind that we are neglecting the effect of the D6 branes)

$$
x^{4} \rightarrow m, \quad x^{6} \rightarrow Q \log \left(\frac{r}{r_{0}}\right)
$$


as $r \rightarrow \infty$. Here $r_{0}$ is some length scale, required on dimensional grounds. Comparing with (4.5), we see that the theory on the NS' brane has the same problem as the M-theory curve of the previous section - there is no solution $x^{4}(r), x^{6}(r)$ to the equations of motion that satisfies (4.6) and intersects the D6 brane at $x^{4}=x^{6}=0$. Therefore, we conclude that even for small $g_{s}$, there is no meta-stable brane configuration satisfying all the desired boundary conditions.

\section{Kink quasi-solutions}

In the previous two sections, we have argued that at $g_{s} \neq 0$ there is no nonsupersymmetric brane configuration corresponding to the meta-stable vacuum of SQCD. However, the question still remains: what happens to the IIA brane configuration of figure 7 when $g_{s}$ is increased? One answer is that it becomes the curve (3.22) $-(3.23)$ with the wrong behavior at infinity. On the other hand, if we fix the boundary conditions at infinity, then the IIA brane configuration must develop an instability somewhere.

In this section, we will analyze this instability in some detail, by constructing a kink "quasi-solution" that satisfies the equations of motion everywhere except along a co-dimension one surface, has the right boundary conditions, and reduces to the metastable configuration as $g_{s} \rightarrow 0$. We will identify a runaway tadpole mode in this solution, which destabilizes it at nonzero $g_{s}$. In the $g_{s} \rightarrow 0$ limit, this tadpole mode is frozen out its kinetic term diverges by a non-perturbative amount.

One can construct these kink quasi-solutions either using the M5 brane action in Mtheory, or using the type IIA NS5 worldvolume action with a D4 spike, that we have used in section 4. In the following two subsections we analyze these two setups and show that their physics is identical. In the last subsection we analyze analytically the runaway mode in the IIA construction.

\subsection{An M-theory kink}

To build a kink solution in M-theory with the right boundary conditions, the simplest thing to do is to patch together the straight-line curve (3.22)-(3.23), which has the correct 
behavior at the D6 brane, together with the solution (3.21), which has the correct behavior at infinity. The result is a family of kink quasi-solutions,

$$
\begin{array}{ll}
x^{6}<L: \quad & \left|x^{8}+i x^{9}\right|= \\
& R \ell_{s}^{2} \mu^{2} e^{-\left(L_{0}-L\right) / 2 N_{c} R} e^{\sqrt{1+(\Delta x / L)^{2}}\left(x^{6}-L\right) / 2 N_{c} R}\left(\frac{\left(1+\sqrt{1+(\Delta x / L)^{2}}\right) x^{6}}{R}\right)^{N_{f} / 2 N_{c}} \\
& x^{4}=\frac{\Delta x}{L} x^{6} \\
x^{6}>L: \quad & \left|x^{8}+i x^{9}\right|=R \ell_{s}^{2} \mu^{2} e^{\left(x^{6}-L_{0}\right) / 2 N_{c} R}\left(\frac{x^{6}+\sqrt{\left(x^{6}\right)^{2}+(\Delta x)^{2}}}{R}\right)^{N_{f} / 2 N_{c}} \\
& x^{4}=\Delta x
\end{array}
$$

parameterized by the position $x^{6}=L$ of the kink. Substituting into the action and integrating, we find after a few technically involved but straightforward steps

$$
S(L)-S\left(L_{0}\right)=\left(\sqrt{L^{2}+(\Delta x)^{2}}-L\right)-\left(\sqrt{L_{0}^{2}+(\Delta x)^{2}}-L_{0}\right)
$$

As expected, this potential has runaway behavior which pushes $L \rightarrow \infty$.

\subsection{A IIA kink}

Since the tadpole found above exists arbitrarily close to $g_{s}=0$ it is also instructive to present the kink quasi-solutions using the IIA NS5 worldvolume action. Starting from the general solution (4.5) and using the same strategy as in the M-theory curve, we obtain

$$
\begin{aligned}
x^{6}<L: & x^{4}=\Delta x+\frac{Q \Delta x}{\sqrt{(\Delta x)^{2}+L^{2}}} \log \frac{r}{r_{k i n k}} \\
x^{6} & =\frac{L}{\Delta x} x^{4} \\
x^{6}>L: \quad x^{4} & =\Delta x \\
x^{6} & =L_{0}+Q \log \frac{r}{r_{0}}
\end{aligned}
$$

where $r_{k i n k}=r_{0} \exp \left(\frac{L-L_{0}}{Q}\right)$ describes the point in $r$ that the two curves meet and $Q$ is defined in (4.4). The curve meets the D6 brane at $r_{D 6}=r_{0} e^{\left(L-L_{0}-\sqrt{(\Delta x)^{2}+L^{2}}\right) / Q}$. Substituting the curve into the NS5 Lagrangian (4.3) and integrating from $r_{D 6}$ to some cutoff, we find the action of the kink:

$$
S=V(L) \equiv Q\left(\sqrt{L^{2}+(\Delta x)^{2}}-L\right)-\frac{1}{2} \exp \left[-2\left(\sqrt{L^{2}+(\Delta x)^{2}}-L+L_{0}\right) / Q\right]
$$


One can check that this potential has no minimum, and leads to runaway behavior $L \rightarrow \infty$. In the limit $Q \rightarrow 0$ the second term is non-perturbatively small and is negligible relative to the first term. The M-theory calculation only gave us the first term in the right hand side of (5.4); thus the second term probably comes from the small mistake we are making in neglecting the effect of the D6 brane.

\subsection{Effective $4 D$ field theory of the kink}

In order to understand the physics of the kink quasi-solution (5.3), it is very instructive to find the effective $4 \mathrm{D}$ field theory that describes its runaway mode. To do that, we promote $L$ to a field, $L=L\left(x^{\mu}\right)$ where $\mu=0,1,2,3$. Then the kink quasi-solution (5.3) depends on $x^{\mu}$ through $L$. The effective $4 \mathrm{D}$ field theory of the kink can be analyzed both using the M-theory kink and the IIA one. They both give the same result. Since the Mtheory calculation involves complicated integrals, and is less clear than the IIA calculation, we only present the latter.

We substitute the kink into the full NS5' action,

$$
S=\int d^{4} x \int d r d \theta \sqrt{\operatorname{det}\left(g_{a b}+g_{s}^{2} F_{a} F_{b}\right)}
$$

and expand up to quadratic order in derivatives of $L\left(x^{\mu}\right)$. The action now involves all of the NS5' worldvolume coordinates

$$
x^{a}, x^{b} \in\left(x^{\mu}, r, \theta\right)
$$

while the induced metric is now $g_{a b}=\partial_{a} x^{M} \partial_{b} x^{M}$, with $M$ running over the space-time coordinates

$$
x^{M} \in\left(x^{\mu}, r, \theta, x^{4}\left(r ; L\left(x^{\mu}\right)\right), x^{6}\left(r ; L\left(x^{\mu}\right)\right)\right)
$$

The result of the expansion is the following $4 \mathrm{D}$ effective action:

$$
S=\int d^{4} x\left[V\left(L\left(x^{\mu}\right)\right)+f\left(L\left(x^{\mu}\right)\right)\left(\partial_{\mu} L\right)^{2}+\ldots\right]
$$

where $V\left(L\left(x^{\mu}\right)\right)$ is defined in (5.4) and

$$
\begin{aligned}
& f(L)= \frac{1}{6} Q \Delta x \sin \theta-e^{-2\left(L_{0}+\Delta x \tan \frac{\theta}{2}\right) / Q}\left(\sin ^{4} \frac{\theta}{2}+\frac{Q^{2}}{8(\Delta x)^{2}} \sin ^{4} \theta\right) \\
&+e^{2\left(L-L_{0}\right) / Q}\left(\sin ^{2} \frac{\theta}{2}-\frac{Q}{4 \Delta x} \sin ^{3} \theta+\frac{Q^{2}}{8(\Delta x)^{2}} \sin ^{4} \theta\right) \\
& \sin \theta \equiv \frac{\Delta x}{\sqrt{(\Delta x)^{2}+L^{2}}}, \quad 0 \leq \theta \leq \frac{\pi}{2}
\end{aligned}
$$


So the coefficient of the kinetic term of $L$ has very different $g_{s} \rightarrow 0$ limits depending on whether $L<L_{0}$ or $L>L_{0}$. When $L>L_{0}$ it diverges due to the exponential factor $e^{2\left(L-L_{0}\right) / Q}$. When $L<L_{0}$ it is regular, since both exponential factors are very small. Therefore, in the $g_{s} \rightarrow 0$ limit, we have a mode which sees the potential (5.4) for $L<L_{0}$, rolls to $L=L_{0}$, and then freezes out because it acquires an infinite kinetic term for $L>L_{0}$. This reproduces exactly the $g_{s}=0$ configuration discussed in section 3 .

As $g_{s}$ starts increasing, the kinetic term for the kink becomes finite everywhere, and the kink runs away to infinity. Even if the kink is not an exact solution, we believe this frozen runaway mode closely approximates the physics of the meta-stable configuration at $g_{s} \neq 0$.

\section{Acknowledgments}

We would like to acknowledge discussions with the participants in the IAS string theory journal club of May 3 2006, where we found the $g_{s}=0$ SUSY-breaking brane configurations in figures 6 and 7. We are indebted to Ken Intriligator for interesting discussions and early collaboration on this project. We would also like to thank David Kutasov, Gregory Moore, Raul Rabadan and Edward Witten, for useful discussions.

The research of IB is supported in part by NSF grant PHY-0503584. The research of EG is supported in part by DOE grant DOEFG03-97ER40546. The research of SH and NS is supported in part by DOE grant DE-FG02-90ER40542. S.H. is the D. E. Shaw \& Co., L. P. Member at the Institute for Advanced Study. The research of DS is supported in part by a Porter Ogden Jacobus Fellowship and by NSF grant PHY-0243680.

\section{Appendix A. Exact Results for Minimal-Area M-theory Curves}

In this appendix, we wish to show how to obtain the exact solutions (3.20) for the non-holomorphic, minimal-area M-theory curve. The action is

$$
A=\int d s \sqrt{\left(V^{-1}+\frac{g^{2}}{4 R^{2} N_{c}^{2}}\right)\left(V\left(1+f^{\prime 2}\right)+g^{\prime 2}\right)}
$$

where for $N_{f}$ coincident D6 branes, the potential takes the form

$$
V=1+\frac{N_{f} R}{\sqrt{f^{2}+s^{2}}}
$$


From this action, we obtain equations of motion for $f$ and $g$ which are second-order differential equations. Using these, we can eliminate $g^{\prime \prime}$ and obtain relatively simple equation involving only $g$ and $g^{\prime}$ :

$$
4 N_{c}^{2} R^{2}\left(g^{\prime 2}\left(\frac{\partial V}{\partial f}-f^{\prime} \frac{\partial V}{\partial s}\right)+2 V^{2} f^{\prime \prime}\right)-g^{2}\left(\left(1+f^{\prime 2}\right) V^{2}\left(\frac{\partial V}{\partial f}-f^{\prime} \frac{\partial V}{\partial s}\right)-2 V^{3} f^{\prime \prime}\right)=0
$$

Solving for $g^{\prime}$ and substituting it back (along with its derivative) into the equations of motion, we find a surprise: $g$ can be eliminated altogether! The resulting equation is:

$$
f^{\prime \prime \prime}\left(\frac{\partial V}{\partial f}-f^{\prime} \frac{\partial V}{\partial s}\right)+f^{\prime \prime}\left(3 f^{\prime \prime} \frac{\partial V}{\partial s}+\left(f^{\prime 2}-1\right) \frac{\partial^{2} V}{\partial f \partial s}+f^{\prime} \frac{\partial^{2} V}{\partial s^{2}}-f^{\prime} \frac{\partial^{2} V}{\partial f^{2}}\right)=0
$$

One can immediately see from this equation that a straight line $\left(f^{\prime \prime}=0\right)$ is always a solution regardless of the form of the function $V$. Indeed, this is expected from the IIA reduction - such a line reduces in type IIA to a D4 in the $x^{4}-x^{6}$ plane, which is always BPS with respect to the D6 branes. Even more, the D4 brane is BPS with respect to an arbitrary distribution of parallel D6 branes, which when lifted to M-theory give rise to an arbitrary $V$. Hence equation (A.4) correctly reproduces the BPS configurations of D4 and D6 branes expected from a IIA analysis. However, these straight line configurations do not give $f \rightarrow m$ as $s \rightarrow \infty$, so they have the wrong boundary conditions at infinity.

To see whether there are any other solutions with the correct boundary conditions, let us focus for simplicity on the case of $N_{f}$ coincident D6 branes. Substituting (A.2) in (A.4), we obtain a nonlinear differential equation for $f$ alone:

$$
3 f^{\prime \prime}\left(f^{\prime \prime} s^{3}-f^{\prime} s^{2}+f^{2} f^{\prime \prime} s-f f^{\prime 2} s+f s+f^{2} f^{\prime}\right)-\left(s^{2}+f^{2}\right)\left(s f^{\prime}-f\right) f^{\prime \prime \prime}=0
$$

In fact, this equation can be integrated in $s$, yielding a second order differential equation:

$$
f^{\prime \prime}=b_{1}\left(\frac{f-s f^{\prime}}{\sqrt{s^{2}+f^{2}}}\right)^{3}
$$

where $b_{1}$ is an integration constant. One can check this, for instance, by substituting it and its derivative into (A.5). We can also introduce

$$
\widetilde{f} \equiv \frac{f}{s}
$$

in terms of which (A.6) becomes

$$
\tilde{f}^{\prime \prime}+\frac{2 \tilde{f}^{\prime}}{s}+\frac{b_{1} s^{2} \tilde{f}^{\prime 3}}{\left(1+\widetilde{f}^{2}\right)^{3 / 2}}=0
$$


Amazingly, this equation can also be integrated in $s$, yielding

$$
\widetilde{f}^{\prime}-\frac{\sqrt{1+\widetilde{f}^{2}}}{s^{2}\left(b_{1} \tilde{f}+b_{2} \sqrt{1+\widetilde{f}^{2}}\right)}=0
$$

This equation can be solved exactly, yielding

$$
f=\frac{b_{2}\left(1-b_{3} s\right)+b_{1} \sqrt{\left(1-b_{3} s\right)^{2}-\left(b_{1}^{2}-b_{2}^{2}\right) s^{2}}}{b_{1}^{2}-b_{2}^{2}}
$$

A straightforward change of variables from $b_{1,2,3}$ to $c_{1,2,3}$ yields the formula (3.20) quoted in the text. 


\section{References}

[1] A. Hanany and E. Witten, "Type IIB superstrings, BPS monopoles, and threedimensional gauge dynamics," Nucl. Phys. B 492, 152 (1997) arXiv:hep-th/9611230.

[2] E. Witten, "Solutions of Four-Dimensional Field Theories Via M-Theory," arXiv:hepth/9703166

[3] A. Giveon and D. Kutasov, "Brane dynamics and gauge theory," Rev. Mod. Phys. 71, 983 (1999) arXiv:hep-th/9802067.

[4] E. Witten, "Branes and the dynamics of QCD," Nucl. Phys. B 507, 658 (1997) arXiv:hep-th/9706109.

[5] K. Hori, H. Ooguri and Y. Oz, "Strong coupling dynamics of four-dimensional N = 1 gauge theories from M theory fivebrane," Adv. Theor. Math. Phys. 1, 1 (1998) arXiv:hep-th/9706082.

[6] A. Brandhuber, N. Itzhaki, V. Kaplunovsky, J. Sonnenschein and S. Yankielowicz, "Comments on the M theory approach to N = 1 SQCD and brane dynamics," Phys. Lett. B 410, 27 (1997) arXiv:hep-th/9706127.

[7] K. Intriligator, N. Seiberg and D. Shih, "Dynamical SUSY breaking in meta-stable vacua," JHEP 0604, 021 (2006) arXiv:hep-th/0602239.

[8] S. Franco and A. M. Uranga, "Dynamical SUSY breaking at meta-stable minima from D-branes at obstructed geometries," JHEP 0606, 031 (2006) arXiv:hep-th/0604136.

[9] V. Braun, E. I. Buchbinder and B. A. Ovrut, "Towards realizing dynamical SUSY breaking in heterotic model building," arXiv:hep-th/0606241.

[10] H. Ooguri and Y. Ookouchi, "Landscape of supersymmetry breaking vacua in geometrically realized gauge theories," arXiv:hep-th/0606061.

[11] V. Braun, E. I. Buchbinder and B. A. Ovrut, "Dynamical SUSY breaking in heterotic M-theory," Phys. Lett. B 639, 566 (2006) arXiv:hep-th/0606166.

[12] T. Banks, "Remodeling the pentagon after the events of 2/23/06," arXiv:hepph/0606313.

[13] S. Ray, "Some properties of meta-stable supersymmetry-breaking vacua in WessZumino models," arXiv:hep-th/0607172.

[14] S. Forste, "Gauging flavour in meta-stable SUSY breaking models," arXiv:hepth/0608036.

[15] A. Amariti, L. Girardello and A. Mariotti, "Non-supersymmetric meta-stable vacua in SU(N) SQCD with adjoint matter," arXiv:hep-th/0608063.

[16] M. Bershadsky, C. Vafa and V. Sadov, "D-Strings on D-Manifolds," Nucl. Phys. B 463, 398 (1996) arXiv:hep-th/9510225. A. M. Uranga, "Brane configurations for branes at conifolds," JHEP 9901, 022 (1999) arXiv:hep-th/9811004. K. Dasgupta and S. Mukhi, "Brane constructions, conifolds and M-theory," Nucl. Phys. B 551, 204 
(1999) arXiv:hep-th/9811139. A. Giveon, D. Kutasov and O. Pelc, "Holography for non-critical superstrings," JHEP 9910, 035 (1999) arXiv:hep-th/9907178.

[17] N. Seiberg, "Electric - magnetic duality in supersymmetric nonAbelian gauge theories," Nucl. Phys. B 435, 129 (1995) arXiv:hep-th/9411149.

[18] M. Schmaltz and R. Sundrum, "N = 1 field theory duality from M-theory," Phys. Rev. D 57, 6455 (1998) arXiv:hep-th/9708015.

[19] K. Hori, "Branes and electric-magnetic duality in supersymmetric QCD," Nucl. Phys. B 540, 187 (1999) arXiv:hep-th/9805142.

[20] H. Ooguri and Y. Ookouchi, "Meta-Stable Supersymmetry Breaking Vacua on Intersecting Branes," arXiv:hep-th/0607183.

[21] S. Franco, I. Garcia-Etxebarria and A. M. Uranga, "Non-supersymmetric meta-stable vacua from brane configurations," arXiv:hep-th/0607218.

[22] S. Elitzur, A. Giveon and D. Kutasov, "Branes and N = 1 duality in string theory," Phys. Lett. B 400, 269 (1997) [arXiv:hep-th/9702014].

[23] G. W. Gibbons and P. Rychenkova, "HyperKaehler quotient construction of BPS monopole moduli spaces," Commun. Math. Phys. 186, 585 (1997) arXiv:hepth/9608085].

[24] N. J. Hitchin, "Polygons and gravitons," In Gibbons, G.W. (ed.), Hawking, S. W. (ed.): Euclidean quantum gravity 527-538.

[25] J. de Boer, K. Hori, H. Ooguri and Y. Oz, "Kaehler potential and higher derivative terms from M theory five-brane," Nucl. Phys. B 518, 173 (1998) arXiv:hepth/9711143.

[26] C. G. Callan and J. M. Maldacena, "Brane dynamics from the Born-Infeld action," Nucl. Phys. B 513, 198 (1998) arXiv:hep-th/9708147.

[27] I. A. Bandos, A. Nurmagambetov and D. P. Sorokin, "The type IIA NS5-brane," Nucl. Phys. B 586, 315 (2000) arXiv:hep-th/0003169]. 